\title{
Endoscopic Endonasal Transoculomotor Triangle Approach for the Resection of a Pituitary Adenoma with Ambient Cistern Extension
}

\author{
Georgios Andrea Zenonos ${ }^{1}$ Eric Wesley Wang ${ }^{2}$ Juan C. Fernandez-Miranda ${ }^{1}$
}

${ }^{1}$ Department of Neurological Surgery, University of Pittsburgh, Pittsburgh, Pennsylvania, United States

2 Department of Otolaryngology, University of Pittsburgh Medical

Address for correspondence Georgios Andrea Zenonos, MD, Department of Neurological Surgery, University of Pittsburgh, 200 Lothrop Street, Suite B400, Pittsburgh, PA 15213-2536,

Center, Pittsburgh, Pennsylvania, United States United States (e-mail: zenonosg@upmc.edu).

J Neurol Surg B 2018;79(suppl S3):S283.

\section{Abstract \\ Keywords \\ - oculomotor triangle \\ - endoscopic endonasal \\ - ambient cistern \\ - extensive pituitary adenoma}

Objectives The current video presents the nuances of the endoscopic endonasal transoculomotor triangle approach for the resection of a pituitary adenoma with extension into the ambient cistern.

Design The video analyzes the presentation, preoperative workup and imaging, surgical steps and technical nuances of the surgery, the clinical outcome, and follow-up imaging.

Setting The patient was treated by a skull base team consisting of a neurosurgeon and an ENT surgeon at a teaching academic institution.

Participants The case refers to a 62-year-old female who presented with vision loss and headaches, and was found to have a pituitary adenoma with extension into the ambient cistern.

Main Outcome Measures The main outcome measures consist of the reversal of the patient symptoms (headaches), the recurrence-free survival based on imaging, as well as the absence of any complications.

Results The patient's headaches improved. There was no evidence of recurrence.

Conclusions The endoscopic endonasal transoculomotor triangle approach is safe and effective for addressing pituitary tumors which extend into the ambient cistern. The link to the video can be found at: https://youtu.be/EBLwEWhohxY.
Conflict of Interest

None.

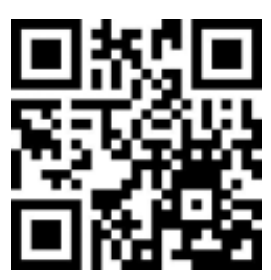

received

October 15, 2017

accepted after revision

December 21, 2017

published online

February 14, 2018

www.thieme.com/skullbasevideos

www.thieme.com/jnlsbvideos

DOI https://doi.org/

10.1055/s-0038-1625942.

ISSN 2193-6331.
๑) 2018 Georg Thieme Verlag KG
Stuttgart · New York

License terms

(c) (1) $\ominus$ (\$) 